OPEN ACCESS

Edited by:

Andrés M. Pérez-Acosta,

Rosario University, Colombia

Reviewed by:

Dalnim Cho,

University of Texas MD Anderson

Cancer Center, United States

Cesare Massimo Cavalera,

Catholic University of the Sacred

Heart, Italy

Cecilia Serena Pace,

University of Genoa, Italy

${ }^{*}$ Correspondence:

Rashid Menhas

menhas.r@yahoo.com

†These authors have contributed equally to this work

Specialty section:

This article was submitted to

Health Psychology,

a section of the journal

Frontiers in Psychology

Received: 16 February 2021

Accepted: 28 June 2021

Published: 16 August 2021

Citation:

Dai J, Sang X, Menhas R, Xu X, Khurshid S, Mahmood S, Weng Y, Huang J, Cai Y, Shahzad B, lqbal W, Gul M, Saqib ZA and Alam MN (2021) The Influence of COVID-19 Pandemic on Physical Health-Psychological Health, Physical Activity, and Overall Well-Being: The Mediating Role of Emotional Regulation.

Front. Psychol. 12:667461. doi: 10.3389/fpsyg.2021.667461

\section{The Influence of COVID-19 Pandemic on Physical Health-Psychological Health, Physical Activity, and Overall Well-Being: The Mediating Role of Emotional Regulation}

Jianhui Dai ${ }^{1+}$, Xuehui Sang ${ }^{1 \dagger}$, Rashid Menhas ${ }^{2 *}$, Xia Xu ${ }^{3}$, Sumaira Khurshid ${ }^{4}$, Sajid Mahmood ${ }^{5}$, Yu Weng ${ }^{1}$, Jiaai Huang ${ }^{6}$, Yuwei Cai ${ }^{6}$, Babar Shahzad ${ }^{7}$, Waseem Iqbal ${ }^{7}$, Maryam Gul ${ }^{8}$, Zulkaif Ahmed Saqib ${ }^{9}$ and Muhammad Nurul Alam ${ }^{10}$

${ }^{1}$ School of Physical Education and Sports, Soochow University, Suzhou, China, ${ }^{2}$ Research Center of Sports Social Sciences, School of Physical Education and Sports, Soochow University, Suzhou, China, ${ }^{3}$ Hubei Key Laboratory of Sport Training and Monitoring, Wuhan Sports University, Wuhan, China, ${ }^{4}$ School of Education and Science, Neijang Normal University, Neijiang, China, ${ }^{5}$ Department of Zoology, Hazara University, Mansehra, Pakistan, ${ }^{6}$ Graduate School, Wuhan Sports University, Wuhan, China, ${ }^{7}$ School of Energy, Soochow University, Suzhou, China, ${ }^{8}$ Department of Applied Psychology, Lahore College for Women University, Lahore, Pakistan, ${ }^{9}$ College of Management, Shenzhen University, Shenzhen, China, ${ }^{10}$ Academy for Research Skills Development (AFRSD), Dhaka, Bangladesh

Background: Highly infectious respiratory disease COVID-19 emerged in Wuhan, China, and spread worldwide. Different measures have been adopted worldwide to contain the COVID-19, and these measures have various impacts on health-related quality of life (HRQoL). This study aimed to assess the impact of the COVID-19 pandemic (CP) and lockdown policy on physical health $(\mathrm{PH})$-psychological health $(\mathrm{PsH})$, physical activity (PA), and overall well-being (OW) in the context of HRQoL, exploring the mediating role of emotional regulation (ER).

Method: The current study was conducted in two provincial cities of China. An online survey was conducted in both the cities to collect the data. After quantifying the data, a total of 2,200 respondents data were analyzed through appropriate statistical techniques.

Results: The study results indicate that $\mathrm{CP}$ was found significantly and negatively related to $\mathrm{PH}(\beta=-0.157, t=9.444, p<0.001)$. A significant relationship was found between $\mathrm{CP}$ and $\mathrm{PsH}(\beta=0.779, t=45.013, p<0.001)$. The third prediction revealed a significant negative relationship between the $\mathrm{CP}$ and $\mathrm{OW}(\beta=-0.080, t=5.261, p<0.001)$. The $\mathrm{CP}$ and PA had a significant negative relationship $(\beta=-0.047, t=3.351, p<0.001)$.

Conclusion: The $\mathrm{PH}, \mathrm{PSH}$, and $\mathrm{OW}$ of the Chinese people were affected due to the $\mathrm{CP}$ and lockdown measures. It is suggested that ER intervention reduces the negative psychological impacts for improving quality of life. ER can function one's sentiments in their social environment effectively for quality of life.

Keywords: COVID-19, lockdown, HRQoL, emotional regulation, physical health, psychological health, physical activity, overall well-being 


\section{INTRODUCTION}

In early December 2019, in Wuhan, Hubei Province, China, a highly infectious respiratory disease, that is, Coronavirus (COVID-19) emerged and extended globally. On March 11, 2020, this newly emerged viral infection was declared a worldwide health emergency by World Health Organization (WHO) (Rogowska et al., 2020). According to the WHO, the COVID19 has spread worldwide, and 213 countries are taking multiple measures to contain the COVID-19 by their governments. On January 23, 2020, the governments implemented various measures, such as the lockout of entire cities, travel warning regulation, and home medical observations to prevent and control the viral transmission (Anna, 2020). Because of the threats of COVID-19 pandemic (CP) to health care systems and society at large, and to reduce the incidence of novel infections and flatten the COVID-19 infection curve, a global mass homeconfinement directive has been implemented in many countries, most of which entail social isolation and quarantine. Social isolation and quarantine can be the main stressors that can lead to emotional distress and other unpredicted mental health and psychological consequences (Hossain et al., 2020). Pandemics have different stages and come in waves with various severe impacts on human health and society. CP is also coming in waves in which the virus pathogen is becoming more dangerous, creating pressure on public health facilities worldwide. Many countries worldwide introduced different policies that include total lockdown, smart lockdown, health monitor system, and quarantine to contain the virus (Alwan et al., 2020). Public health experts review the CP-related lockdown policies (LPs) daily to ensure public safety (Al Zobbi et al., 2020; Plümper and Neumayer, 2020).

Studies have reported loneliness, anxiety, boredom, anger, denial, depression, insomnia, harmful substance use, despair, self-harm, and suicides in quarantined individuals ( $\mathrm{Li}$ et al., 2020; Wang et al., 2020). Furthermore, COVID-19 physical symptoms (such as cough, hypoxia, and fever) along with side effects of recommended medicines (corticosteroids) may lead to more psychological distress and anxiety (Wang et al., 2020). Researchers reported that various psychiatric disorders could be found in individuals, for instance, anxiety disorders, selfblame, guilt, post-traumatic stress disorder, depressive disorders, delirium, somatic symptoms, panic disorder, psychosis, and even suicide (Goyal et al., 2020). Furthermore, our findings are similar in the context of emotional regulation (ER) with the studies of Li et al. (2021) and Cheng et al. (2006), which were carried out among the Chinese population and concluded that socio-behavioral restrictions are negatively associated with the health-related quality of life (HRQoL). There is also a negative impact on emotions on time spent under quarantine measures. It is consistent with previously published work on the mental health effects of the lockout of COVID-19 (OzamizEtxebarria et al., 2020). Several studies reported adverse impacts, such as depression, loneliness, anxiety, and post-traumatic stress due to the $\mathrm{CP}$. The suddenly declared pandemic has drastic negative effects on every segment of human society in the socio-psychological and physical paradigm. Muzi et al. (2021) conducted a study on Italian adolescents and reported that the teenagers might have used social media disorder symptoms to express CP adverse effects. Teenagers during pandemic showed lower internalizing but higher other issues (such as excessive drinking and self-destructive behaviors) and more problematic social media usage than pre-pandemic samples.

\section{COVID-19 Lockdown-Health and Well-Being}

HRQoL, participation in physical activity (PA), and perceived mental stress among Chinese adults are significantly related to the CP. Physical inactivity and sedentary sitting time have been increased during home confinement among Chinese people. Long-term sitting has also proved to negatively impact wellbeing and quality of life (Qi et al., 2020). Lockdown policy (LP) implementation across China has generated many sociopsychological problems for the Chinese people in every segment of their lives. During the period of lockdown, people were confined to their homes to contain the pathogen. Domestic confinement has a long-lasting psychological and well-being effect. Chinese people were confronted by anger, boredom, and loneliness during home confinement, and psychological problems, such as depression, stress, and anxiety increased (Duan and Zhu, 2020). Mental health and quality of life among Chinese adults have been impacted negatively by the CP (Zhang and Ma, 2020). Home isolation has adverse socio-psychological effects on physical and mental health. Long-term isolation causes negative feelings, cognitive decline, and discomfort (Hawkley and Capitanio, 2015). The daily routine and lifestyle of Chinese citizens would inevitably be interrupted by restrictions on travel and outdoor leisure. Individuals were also less physically involved, more sedentary, and more depressed, which may pose severe protection and well-being risks (Chen et al., 2020). HRQoL has been affected due to the socio-psychological impacts of COVID-19 and caused a severe threat to global public health (Tsamakis et al., 2020). People with personality disorders may be particularly vulnerable to negative psychological impacts of the CP. ER skills appear to be a potential target for therapies targeted at reducing negative consequences (Velotti et al., 2021a,b).

\section{COVID-19-Emotional Regulation for Health and Well-Being}

Emotions refer to an event-focused affective state, an intricate pattern of the reaction involving elements of experience, actions, and physiology (Sander et al., 2013). The advent of the CP can exacerbate these two psychological aspects and make people feel endangered. People interpret risk cognitively and respond to it emotionally. Risk beliefs are often the source of negative emotions and psychological distress (Leppin and Aro, 2009). The social and emotional reaction to the epidemic of COVID-19 is multidimensional. Furthermore, it depends not just on external factors but also on personal and innate components (Brooks et al., 2020). Emotions and feelings play a vital role in response to the sudden phenomena and reshape our understanding about how to cope with the negative impacts of different situations on our socio-psychological and health-related QoL. Emotion 
regulation is helpful in the maintenance of health behavior during the CP. Positive health behavior through ER is a catalyst for PA and psychological well-being (Julie, 2020). In empirical models of well-being, human growth, interpersonal processes, psychopathology, and decision making, emotions play a pivotal role (Ekman and Davidson, 1994; Saarni, 2008). Previous pandemics, such as Ebola, H1N1, and SARS evidence show that public emotional response is associated with risk perception (Yang, 2016). During the early stages of the COVID19 outbreak in China, a nationwide survey found that $\sim 27.9 \%$ of the participants had depression symptoms, and $31.6 \%$ had anxiety symptoms, which further leads to emotional sensitivity (Shi et al., 2020). ER is an effective way to maintain healthy behavior in particular circumstances. ER processes have long been known as a trans-diagnostic factor in various psychological problems (Sloan et al., 2017). ER theories indicate that the ability to control one's own emotions is necessary for psycho-social health (Gross and Munoz, 1995). Emotional stability requires explicit or implicit attempts to control the emotional perception, expression, length, and magnitude (Gross, 1998). A significant factor in raising or decreasing the risk of poor physical and $\mathrm{PsH}$ should be controlling feelings when people face the challenges of the pandemic (Low et al., 2020). PA is a catalyst for healthy living and plays a pivotal role in preventing different diseases (Saqib et al., 2020).

\section{Statement of the Study}

Significant adverse impacts have been observed due to the CP, which undermines the overall health and well-being. Preventive measures, such as home isolation during mandatory lockdown to contain the pathogen or the virus are used effectively to manage socio-economic life safely (Holmes et al., 2020). Regulation of emotion is significant in maintaining psychological and physical health $(\mathrm{PH})$ during home isolation and lockdown during the pandemic period. People use emotions to add sense to their interactions and organize themselves and the people they communicate with (Duy and Yildiz, 2014). ER is also a vital principle that allows people to manage their emotional states and maintain healthy relationships with their environment (Gross, 2013). The CP created a fearful and panicked environment that has negative socio-psychological impacts on human lives. Management and regulation of emotions are the best ways to keep positive attitude and behavior toward a healthy quality of life. The pandemic created a crisis across the globe, and emotions are vital to human lives, which give direction to maintain sociopsychological and $\mathrm{PH}$ well-being. This study aimed to assess the impact of the CP and LP on PH-psychological health ( $\mathrm{PsH}), \mathrm{PA}$, and overall well-being (OW) in the context of HRQoL, exploring the mediating role of ER. HRQoL through a mediating role of ER during $\mathrm{CP}$ explored $\mathrm{PH}, \mathrm{PsH}, \mathrm{PA}$, and OW. Every person is experiencing COVID-19 in various ways and has different emotional reactions to manage OW. Regulation of emotions applies to people controlling their emotional perceptions and altering emotional expressions in the face of extreme or negative feelings, intentional behavioral improvement, and regulation of affective states (Leahy et al., 2011). It is especially true for those who have low self-control levels in maintaining their emotions and have drawn attention to reliance on others to manage their negative emotions. For persons who tend to emotional control issues feeling lonely may be extremely motivating. Humans may be able to express themselves based on the information they are processing through emotions. The grand theory of Aristotle proposed that emotions were sentiments associated with happiness or suffering, such as wrath, joy, fear, love, hatred, desire, and confidence (Bound, 2018). ER to negate the negative emotions and prevent psychological issues is the best way among the masses during the CP (Velotti et al., 2021a,b). The relationship between different variables of the study has been shown in Figure 1. The ER strategies have significant impacts on $\mathrm{PH}$ and $\mathrm{PsH}$. ER and physical health well-being have an essential association under particular circumstances. The CP is posing a unique circumstance globally, and people are facing numerous kinds of challenges in every segment of life. In the paradigm of COVID-19, examining the relationship between ER, $\mathrm{PH}, \mathrm{PsH}, \mathrm{PA}$, and $\mathrm{OW}$ have vital implications for health and routine life functioning.

\section{MATERIALS AND METHODS}

\section{Study Locale}

The present study was conducted in two cities of China; Wuhan, Hubei Province, and Suzhou, Jiangsu Province, as shown in Figure 2. The study upheld the standards of the World Medical Helsinki Policy. Therefore, the Ethical Committee of Soochow University, Suzhou, Jiangsu and Wuhan Sports University, Wuhan, Hubei, approved the study.

\section{Participants}

The current study population was Wuhan, Hubei Province, and Suzhou, Jiangsu Province city residents $(+18)$ in the cities during the lockdown period. A convenience sampling method was used to collect the data by conducting an online survey in both the cities. A total of 2,280 respondents replied to the online survey questionnaire. After quantifying the data, a total of 2,200 respondents from Suzhou $(1,034)$ and Wuhan $(1,166)$ were included for the final data analysis. The answers of the 80 respondents were excluded because of incomplete information. According to the table statistics, most of the survey participants ( $\sim 53.0 \%$ ) lived in the Wuhan city, Hubei Province, where the first lockdown was implemented due to the severity of the CP and $\sim 47.0 \%$ lived in Suzhou city, Jiangsu Province, during the lockdown period.

\section{Instrument and Data Collection}

The impacts of CP and LPs were assessed in the context of HRQoL in association with ER, $\mathrm{PH}, \mathrm{PsH}, \mathrm{PA}$, and OW. An online questionnaire survey method was used to collect the primary data from the targeted population in both Wuhan and Suzhou cities. The survey method was used to collect the primary data in the current study. The questionnaire was developed after reviewing the variables related to previous research studies regarding SARS and influenza outbreaks (Rubin et al., 2010). The questionnaire was pre-tested in both cities (Wuhan and Suzhou) targeted population before conducting the final survey for data 

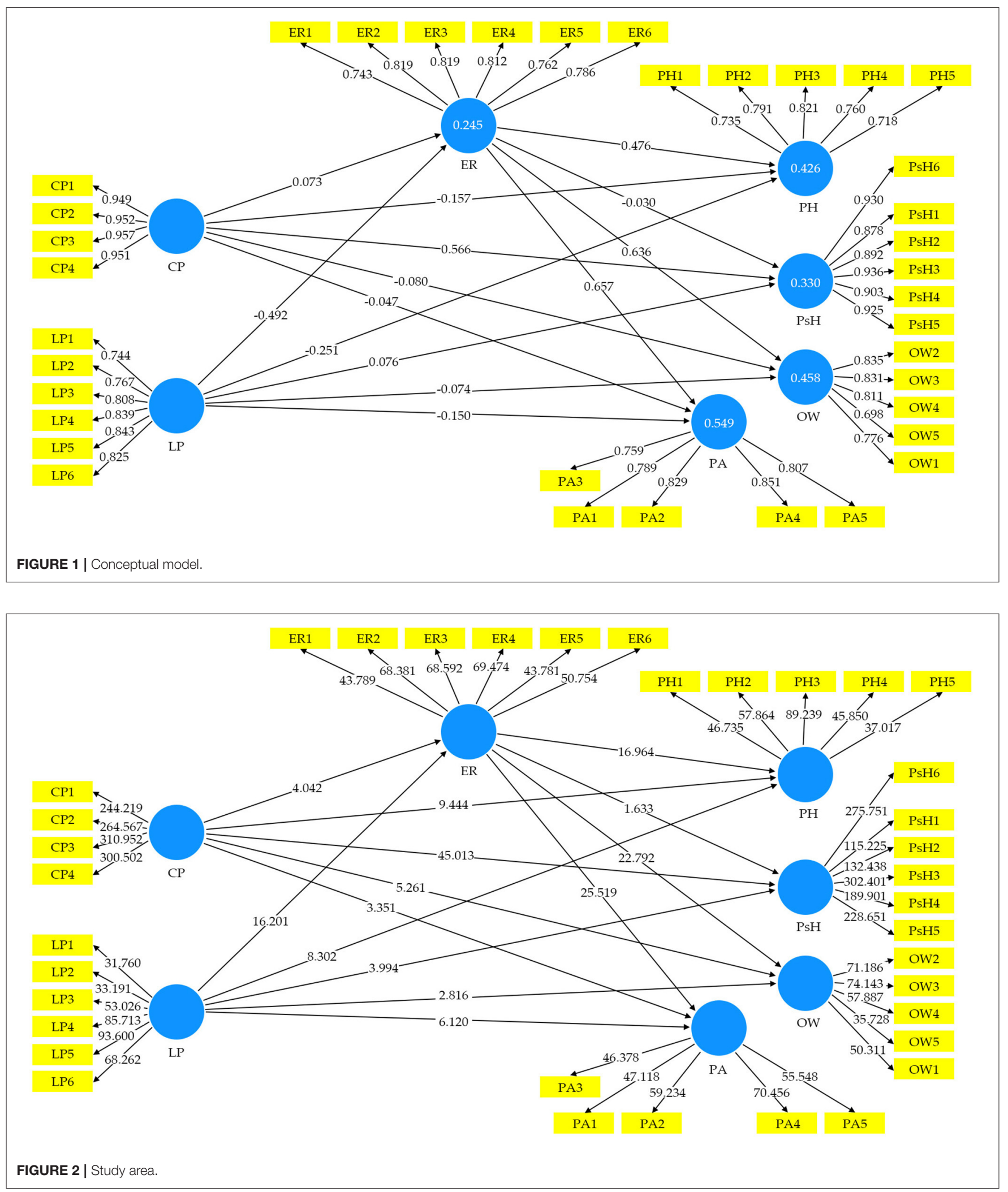

collection. After pre-testing the questionnaire, some questions were amended and improved the wording of the comprehensive questionnaire for getting the best response rate from the study participants. An online survey was conducted in both Wuhan, Hubei Province, and Suzhou, Jiangsu Province cities from July 9 to August 10, 2020, to evaluate the CP and LPs and the HRQoL. 
TABLE 1 | Demographic characteristics of the survey participants.

\begin{tabular}{|c|c|c|c|c|}
\hline City wise demographics & Category & Suzhou $(N=1,034)$ & Wuhan $(N=1,166)$ & Overall $(N=2,200)$ \\
\hline \multirow[t]{6}{*}{ Age (years) } & $18-24$ & $316(30.56 \%)$ & $514(44.08 \%)$ & $830(37.73 \%)$ \\
\hline & $25-34$ & $172(16.63 \%)$ & 21 (1.80\%) & $193(8.77 \%)$ \\
\hline & $35-44$ & $253(24.47 \%)$ & 137 (11.75\%) & 390 (17.73\%) \\
\hline & $45-54$ & 199 (19.25\%) & 215 (18.44\%) & $414(18.82 \%)$ \\
\hline & $55-64$ & 77 (7.45\%) & 59 (5.06\%) & $136(6.18 \%)$ \\
\hline & 65 or older & 17 (1.64\%) & 220 (18.87\%) & 237 (10.77\%) \\
\hline \multirow[t]{4}{*}{ Gender } & Male & 503 (48.65\%) & $583(50.0 \%)$ & 1,086 (49.36\%) \\
\hline & Female & 524 (50.68\%) & 572 (49.05\%) & 1,096 (49.82\%) \\
\hline & Others & $2(0.19 \%)$ & $3(0.26 \%)$ & $5(0.23 \%)$ \\
\hline & Prefer not to answer & $5(0.48 \%)$ & 8 (0.69\%) & $13(0.59 \%)$ \\
\hline \multirow[t]{5}{*}{ Marital status } & Single (never married) & 400 (38.68\%) & $518(49.57 \%)$ & $918(41.73 \%)$ \\
\hline & Married, or in a domestic partnership & $595(57.54 \%)$ & $512(43.91 \%)$ & 1,107 (50.32\%) \\
\hline & Widowed & $11(1.06 \%)$ & $97(8.32 \%)$ & 108 (4.91\%) \\
\hline & Divorced & $22(2.13 \%)$ & 36 (3.09\%) & $58(2.64 \%)$ \\
\hline & Separated & $6(0.58 \%)$ & $3(0.26 \%)$ & $9(0.41 \%)$ \\
\hline \multirow[t]{5}{*}{ Education } & Less than high school degree & 137 (13.25\%) & 354 (30.36\%) & $491(22.32 \%)$ \\
\hline & High School & 197 (19.05\%) & $13(1.11 \%)$ & $210(9.54 \%)$ \\
\hline & Associate degree & $143(13.83 \%)$ & $180(15.44 \%)$ & $323(14.68 \%)$ \\
\hline & Bachelor's degree & 167 (16.15\%) & 414 (35.51\%) & $581(26.41 \%)$ \\
\hline & Graduate degree & 390 (37.72\%) & 205 (17.58\%) & 595 (27.04\%) \\
\hline
\end{tabular}

The questionnaire was based on closed-ended 5-point Likert scale questions regarding the respondents' demographic information, CP, COVID-19 LP, ER, PH, PsH, participation in PA, and OW during the COVID-19 lockdown period. An informed consent received from all the study participants after informing about the purpose of the study. The researchers performed the quality check (accuracy, relevancy, and completeness) of the data collected anonymously. It was guaranteed to all study participants that data would be used only for research.

\section{Conceptualization of Variables and Measurement}

After reviewing the relevant literature and studies conducted by Sang et al. (2021) and Lin et al. (2020) in the socio-economic and CP perspective, the current study included living place (Suzhou, Wuhan); gender (male, female, others, and prefer not to answer); age $(18-24,25-34,35-44,45-54,55-64$, and 65 years or older); education (less than high school degree, high school degree, associate degree, bachelor's degree, and graduate degree); marital status (single-never married, married, or in a domestic partnership, widowed, divorced, and separated); and employment status (employed full time-including self-employed or homemaker, employed part-time-including self-employed or homemaker, unemployed, student, retired, and unable to work); and annual household income before taxes (Pre-COVID-19). The survey participants reported all the demographic variables used in the study. The CP affects everyday life, movements, trade, and business activities from local to global, which further impacts socio-economic lives of people (Haleem et al., 2020).

The CP and LP were used as independent variables in the current study. The CP was assessed by asking questions about risk perception and belief about the pandemic that emerged in
Wuhan. The questions were based upon the belief about that how the COVID-19 emerged (Due to climate change, the CP created fear and anxiety, the belief that the COVID-19 is a threat to humanity, and the importance have health and well-being as a top priority in everyone's life after COVID-19). As a result, the lockdown was initially imposed to contain the transmission of the pandemic. Under the lockdown measure, several interventions were introduced among the general population for physical health well-being protection. In the present study, questions were asked about the LP interventions (stay at home, social distancing, wearing a facemask, wash hands with sanitizer, quarantine and avoid the areas where the pandemic is severe).

ER is the mediator variable according to the study objective. The questions related to ER in the current study were based upon the coping strategy aspects. The study participants were asked to report the strategies or steps (getting comfort and understanding from someone, use the substance to make myself feel better, accept the reality of fact and learned to live with it safely, maintaining positive thinking, to do physical exercise to release stress and anxiety and look for creative ways to alter the problematic situation) which they used during the lockdown period to regulate the emotions for psychological, physical, and overall health well-being. Infectious disease outbreaks are one of the most daunting conditions to deal with emotionally. Physical and emotional well-being of individuals is jeopardized as they must plan for an uncertain event. Since there is no definite time limit for the conclusion of infectious disease outbreaks, people feel at risk all the time (Bavel et al., 2020).

The dependent variables in this study were $\mathrm{PH}, \mathrm{PsH}, \mathrm{PA}$, and OW during the CP lockdown. The question statements under the Likert scale for each dependent variable is based on the $\mathrm{PH}$ (during the COVID-19: Have you maintained personal 
hygiene for disease prevention? Have you been leading an active lifestyle during the COVID-19 lockdown? Did you have a healthy diet during the pandemic lockdown? During COVID19, have you maintained physical fitness? and During COVID-19, did you have a normal sleep of $8 \mathrm{~h}$ ?); PsH (During COVID19 lockdown, have you experienced anxiety, bipolar disorder, insomnia, substance abuse or addiction, depression, and mental stress?); OW (During the CP, your PH remained stable, your PsH remained stable, your lifestyle remained active, your financial situation remained stable and your emotional health remained stable); and PA (use PA to cope with the health maintenance difficulties you faced, encourage others, including your family members, to do physical activities, PA levels during the lockdown period decrease, PA levels during the lockdown period increase and your PA levels during the lockdown period were almost the same). Thus, HRQoL is based on physical, psychological, and OW. Therefore, HRQoL is a multidimensional paradigm in public health and is based on various aspects, such as $\mathrm{PH}, \mathrm{PsH}$, and PA (Sitlinger and Zafar, 2018).

\section{Statistical Analysis}

For analyzing the collected data for this study, the Smart-PLS 3.2.9 and SPSS 23 software were used (Ringle et al., 2015). The statistical analysis was based on two parts; univariate and multivariate. Under the univariate analysis, the demographic information of the survey participants was analyzed. And under the multivariate analysis, the structural equation model (SEM) technique was applied to examine the relationship between the study variables. Two-step techniques were used under the SEM for analyzing the data. The first step was the measurement model for checking construct validity, reliability, and convergent validity $(\mathrm{CV})$. In the second step, the structural model was developed to test the hypothesis (Anderson and Gerbing, 1992; Hair et al., 2017). A robust, scalable, and advanced method for creating a significant statistical model is the Smart-PLS research design. The function of the Smart PLS-SEM helps achieve the intended objective (Abbas et al., 2019). The SEM in this study is based on six observed variables, as shown in Figure 1 (conceptual model), to assess the HRQoL. The CP and LP are the independent variables, while ER is considered a mediator variable. Furthermore, $\mathrm{PH}, \mathrm{PsH}, \mathrm{PA}$, and $\mathrm{OW}$ are considered as dependent variables.

\section{RESULTS}

\section{Univariate Analysis \\ Demographic Characteristics of the Survey \\ Participants $(\mathrm{N}=2,200)$}

Table 1 shows the demographic characteristics of the survey participants. The age statistics show that majority of the survey participants $(\sim 37.73 \%)$ belonged to the age group of $18-24$ years, $\sim 18.82 \%$ belonged to the age group of $45-54$ years, $\sim 17.73 \%$ belonged to the age group of $35-44$ years, $\sim 10.77 \%$ belonged to the age group of +65 years, and $\sim 8.77 \%$ belonged to the age group of $25-34$ years, while only $\sim 6.18 \%$ belonged to the age group of 55-64 years. In the context of gender distribution, the majority of the survey participants $(\sim 49.82 \%)$ were female, with a slight difference of $\sim 49.36 \%$ being male, and only $\sim 0.59 \%$ preferred not to answer about their gender identity. In comparison, only $\sim 0.23 \%$ were others. Table statistics show that majority of the survey participants $(\sim 50.32 \%)$ were married or in a domestic partnership, $\sim 41.73 \%$ were single (never married), $\sim 4.91 \%$ were widowed, and $\sim 2.64 \%$ were divorced. In comparison, only $\sim 0.41 \%$ were separated. The educational background shows that the majority of the survey participants (27.04\%) had a graduate degree, $\sim 26.41 \%$ had a bachelor degree, $\sim 22.32 \%$ had less than high school degree, $\sim 14.68 \%$ had an associate degree, and $\sim 9.54 \%$ had high school level education.

\section{Multivariate Analysis}

\section{Assessment of the Measurement Model}

We examined the internal consistency reliability, $\mathrm{CV}$, and discriminant validity (see Table 3). Cronbach's alpha and composite reliability were utilized to evaluate the internal consistency of the measures used, which ranged from 0.823 to 0.966 and 0.876 to 0.975 , respectively, thus surpassing the 0.70 cutoff in all the cases. For CV, the factor loadings of all the items and the average variance extracted (AVE) were conducted. CV was confirmed because loading of all the items was more than 0.6 , and the AVE for all the constructs was more than 0.5 thresholds (Hair et al., 2017). All the mentioned results are presented in Table 2 and Figure 3.

\section{Discriminant Validity}

There are various approaches to determine discriminant validity, such as Fornell Lacker and Hetro Trait-Mono Trait (HTMT). Fornell Lacker is the first criterion that needs to confirm for discriminant validity. According to this process, the value of the square root of AVE of one construct must be higher than the value of inter-correlations between the constructs. This is because a construct must represent more variance with its items than others in the model. As depicted in Table 3, the square roots of the AVE of all constructs are more significant than their corresponding inter-correlation values. Henseler et al. (2016) proposed the HTMT method regarding discriminant validity, which confirms discriminant validity between each pair of variables. Table 4 shows that the HTMT values are below the threshold of 0.90 .

\section{Assessment of the Structural Equation Model}

According to Chin (2010), the structural model represents the theoretical model to evaluate the inner path model with structural equations. For the evaluation of the SEM in this research, the essential criteria used were path coefficient $(\beta)$, coefficient of determination (R2) for an endogenous variable, effect size (f2), prediction relevance (q2), and multicollinearity (inner VIF) (Tenenhaus et al., 2005; Henseler et al., 2009; Götz et al., 2010). The threshold value and description for each benchmark are shown in Table 5 of the SEM. Table 5 presents the findings related to our direct hypotheses as well; in support of the first prediction, $\mathrm{CP}$ was significantly and negatively related to $\mathrm{PH}(\beta$ $=-0.157, t=9.444, p<0.001$, Table 6). Similarly, a significant relationship between second prediction $\mathrm{CP}$ and $\mathrm{PsH}$ was found ( $\beta=0.779, t=45.013, p<0.001)$. For the third prediction 
TABLE 2 | Construct validity and reliability $(N=2,200)$.

\begin{tabular}{|c|c|c|c|c|c|}
\hline Constructs & Items & FL & CA & CR & AVE \\
\hline \multirow[t]{5}{*}{ COVID-19 pandemic } & $\mathrm{CP}$ & & 0.966 & 0.975 & 0.907 \\
\hline & CP1 & 0.949 & & & \\
\hline & CP2 & 0.952 & & & \\
\hline & CP3 & 0.957 & & & \\
\hline & $\mathrm{CP} 4$ & 0.951 & & & \\
\hline & LP2 & 0.767 & & & \\
\hline & LP3 & 0.808 & & & \\
\hline & LP4 & 0.839 & & & \\
\hline & LP5 & 0.843 & & & \\
\hline & LP6 & 0.825 & & & \\
\hline & ER3 & 0.819 & & & \\
\hline & ER4 & 0.812 & & & \\
\hline & ER5 & 0.762 & & & \\
\hline & ER6 & 0.786 & & & \\
\hline \multirow[t]{6}{*}{ Physical health } & $\mathrm{PH}$ & & 0.823 & 0.876 & 0.587 \\
\hline & $\mathrm{PH} 1$ & 0.735 & & & \\
\hline & $\mathrm{PH} 2$ & 0.791 & & & \\
\hline & $\mathrm{PH} 3$ & 0.821 & & & \\
\hline & $\mathrm{PH} 4$ & 0.760 & & & \\
\hline & PH5 & 0.718 & & & \\
\hline Psychological health & $\mathrm{PsH}$ & & 0.959 & 0.967 & 0.830 \\
\hline \multirow[t]{6}{*}{ Physical activity } & PA & & 0.867 & 0.904 & 0.652 \\
\hline & PA1 & 0.789 & & & \\
\hline & PA2 & 0.829 & & & \\
\hline & PA3 & 0.759 & & & \\
\hline & PA4 & 0.851 & & & \\
\hline & PA5 & 0.807 & & & \\
\hline \multirow[t]{6}{*}{ Overall well-being } & OW & & 0.850 & 0.893 & 0.627 \\
\hline & OW1 & 0.776 & & & \\
\hline & OW2 & 0.835 & & & \\
\hline & OW3 & 0.831 & & & \\
\hline & OW4 & 0.811 & & & \\
\hline & OW5 & 0.698 & & & \\
\hline
\end{tabular}

FL, Factor Loadings; CA, Cronbach's Alpha; CR, Composite Reliability; AVE, Average Variance Extracted; CP, COVID-19 Pandemic; LP, Lockdown Policy; ER, Emotional Regulation; PH, Physical Health; PSH, Psychological Health; PA, Physical Activity; OW, Overall Well-being.

(H3), the statistical analysis revealed that there is a significant negative relationship between $\mathrm{CP}$ and OW $(\beta=-0.080, t=$ 5.261, $p<0.001)$. The fourth hypothesis (H4), the statistical analysis, revealed that there is a significant negative relationship between CP and PA ( $\beta=-0.047, t=3.351, p<0.001)$. Similarly, the other four paths, that is, LP and PH, LP and PsH, LP and 
TABLE 3 | Discriminant validity: Fornell Larcker $(N=2,200)$.

\begin{tabular}{lcccccc}
\hline Constructs & CP & ER & LP & OW & PA & PH \\
\hline CP & $\mathbf{0 . 9 5 2}$ & & & & & \\
ER & 0.057 & $\mathbf{0 . 7 9 1}$ & & & & \\
LP & 0.032 & -0.490 & $\mathbf{0 . 8 0 5}$ & & & \\
OW & -0.046 & 0.668 & -0.388 & $\mathbf{0 . 7 9 2}$ & & $\mathbf{0 . 8 0 8}$ \\
PA & -0.014 & 0.727 & -0.473 & 0.685 & 0.593 & $\mathbf{0 . 7 6 6}$ \\
PH & -0.138 & 0.591 & -0.490 & 0.553 & -0.094 & -0.122 \\
PSH & 0.567 & -0.035 & 0.109 & -0.138 & 0.911 \\
\hline
\end{tabular}

CP, COVID-19 Pandemic; ER, Emotion Regulation; LP, Lockdown Policy; OW; Overall well-being; PA, Physical Activity; PH, Physical Health; PsH, Psychological Health.

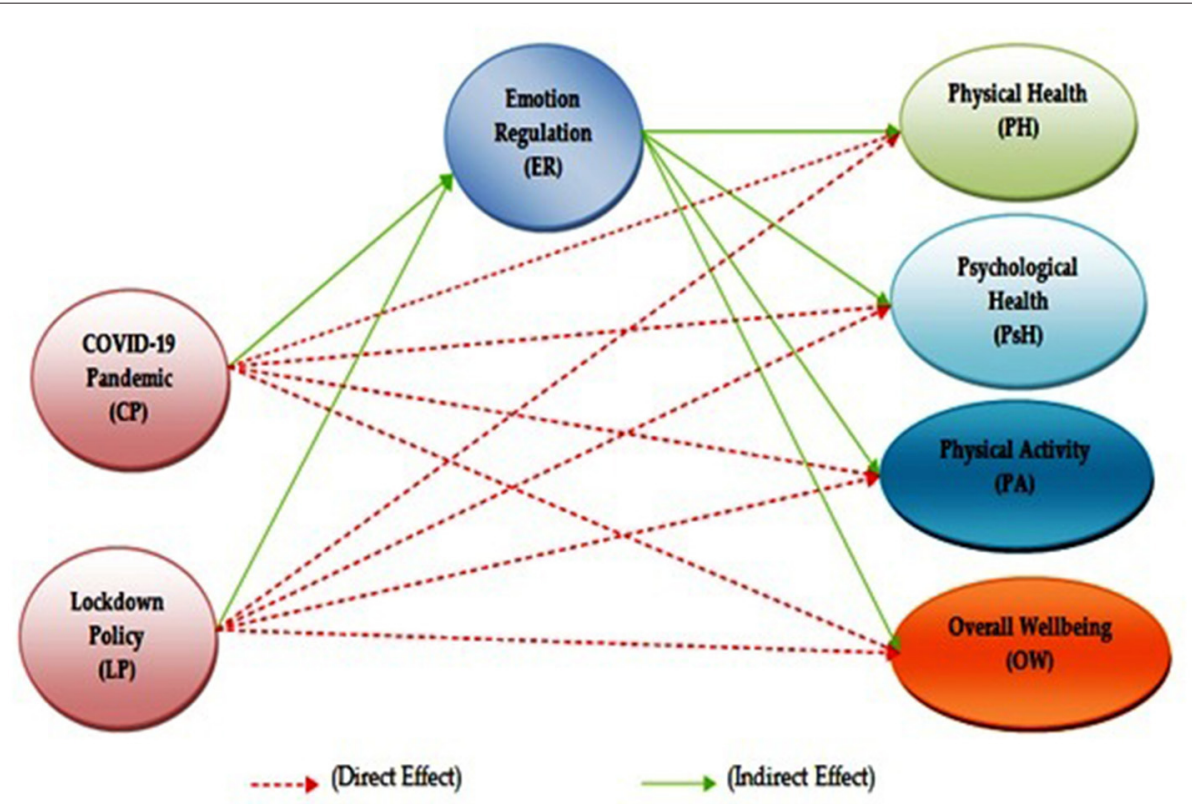

FIGURE 3 | Factor loadings, path coefficient, and R-square result (PLS-algorithm).

TABLE 4 | Discriminant validity (HTMT method) $(N=2,200)$.

\begin{tabular}{|c|c|c|c|c|c|c|c|}
\hline Items & CP & ER & LP & OW & PA & PH & PsH \\
\hline \multicolumn{8}{|l|}{$\mathrm{CP}$} \\
\hline ER & 0.070 & & & & & & \\
\hline LP & 0.071 & 0.537 & & & & & \\
\hline OW & 0.060 & 0.771 & 0.435 & & & & \\
\hline PA & 0.039 & 0.825 & 0.527 & 0.803 & & & \\
\hline $\mathrm{PH}$ & 0.150 & 0.693 & 0.555 & 0.656 & 0.701 & & \\
\hline $\mathrm{PsH}$ & 0.586 & 0.052 & 0.118 & 0.155 & 0.105 & 0.133 & \\
\hline
\end{tabular}

CP, COVID-19 Pandemic; Emotion Regulation; LP, Lockdown Policy; OW, Overall Wellbeing; PA, Physical Activity; PH, Physical Health; PsH, Psychological Health.

OW, and LP and PA, were statistically significant with negative relationships as their $p$-values were $<0.05$. Therefore, the $\mathrm{H} 1$ to H8 was supported, which is presented in Table 6 and Figure 4.

Furthermore, the mediating effect of EC among the mediating path, such as $\mathrm{CP}->\mathrm{ER}->\mathrm{PH}, \mathrm{CP}->\mathrm{ER}->\mathrm{OW}, \mathrm{CP}->\mathrm{ER}$ $->$ PA, LP -> ER -> PH, LP -> ER -> OW, and LP -> ER ->
PA were found statistically significant as the $t$-values were higher than 1.96 and the $p$-values were $<0.05$. Besides the confidence interval results for those relationships, LL and UL were negative values (i.e., " 0 " not in between), which also confirmed the mediation effect. Furthermore, all the mediation effects were found to be of partial mediation as their direct relationships were 
TABLE 5 | Assessment of structural equation model $(N=2,200)$.

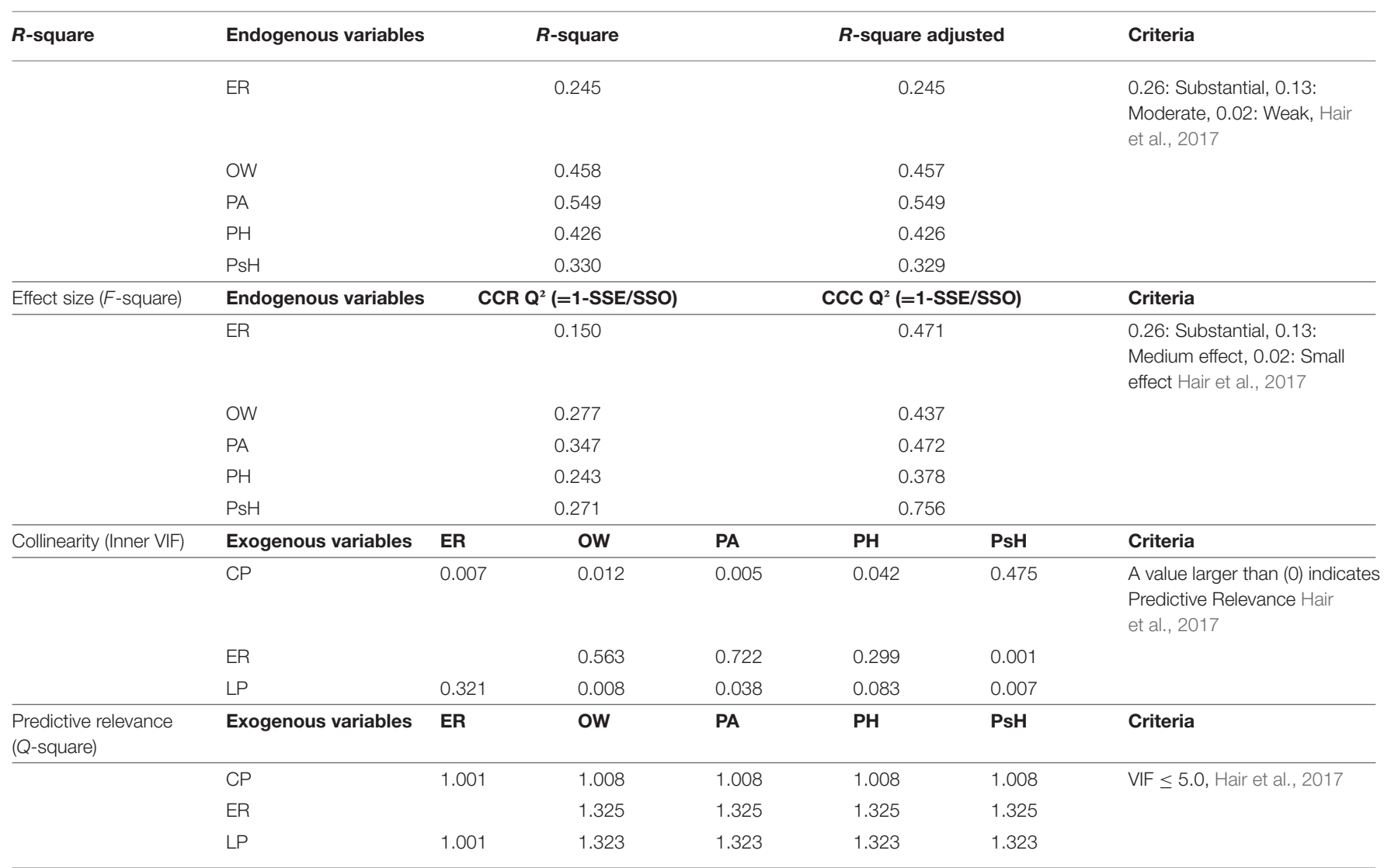

ER, Emotion Regulation; OW, Overall Wellbeing; PA, Physical Activity; PH, Physical Health; PsH, Psychological Health; CP, COVID-19 Pandemic; LP, Lockdown Policy.

TABLE 6 | Path coefficient (direct effect) result $(N=2,200)$.

\begin{tabular}{|c|c|c|c|c|c|c|}
\hline Hypotheses & Original sample (O) & Sample mean (M) & SD & $T$ & $\boldsymbol{P}$-values & Decision \\
\hline $\mathrm{CP}->\mathrm{PH}$ & -0.157 & -0.158 & 0.017 & 9.444 & 0.001 & Significant \\
\hline $\mathrm{CP}->\mathrm{PsH}$ & -0.779 & -0.566 & 0.013 & 45.013 & 0.001 & Significant \\
\hline $\mathrm{CP}->\mathrm{OW}$ & -0.080 & -0.080 & 0.015 & 5.261 & 0.001 & Significant \\
\hline $\mathrm{CP}->\mathrm{PA}$ & -0.047 & -0.046 & 0.014 & 3.351 & 0.001 & Significant \\
\hline $\mathrm{LP}->\mathrm{PH}$ & -0.251 & -0.250 & 0.030 & 8.302 & 0.001 & Significant \\
\hline $\mathrm{LP}->\mathrm{PsH}$ & -0.076 & -0.077 & 0.019 & 3.994 & 0.001 & Significant \\
\hline LP $->$ OW & -0.074 & -0.072 & 0.026 & 2.816 & 0.005 & Significant \\
\hline$L P->P A$ & -0.150 & -0.151 & 0.025 & 6.120 & 0.001 & Significant \\
\hline
\end{tabular}

CP, COVID-19 Pandemic; LP, Lockdown Policy; PH, Physical Health; PsH, Psychological Health; OW, Overall Wellbeing; PA, Physical Activity.

also significant. However, two mediation paths, such as CP -> ER -> PsH and LP - > ER -> PsH, revealed no significant mediation as their $p$-values were higher than 0.05 and zero " 0 " in between LL and UL. All the results are presented in Table 7 and Figure 4.

\section{DISCUSSION}

In the current study, we tried to explore the mediating role of ER. Furthermore, we assessed the impact of the CP and LP on $\mathrm{PH}-\mathrm{PsH}, \mathrm{PA}$, and $\mathrm{OW}$ in the context of HRQoL. The Chinese government implemented strict measures for the safety of the Chinese people to contain the COVID-19 virus. Those measures have significant impacts on the HRQoL of the Chinese people.

The findings related to our first hypothesis confirmed our first prediction that $\mathrm{CP}$ was found significantly and negatively associated with $\mathrm{PH}$. These findings are in line with the previous studies that reported the $\mathrm{CP}$ and the LPs negatively impacting the HRQoL during the COVID-19 epidemic in Morocco (Azizi et al., 2020). The CP affected PH by increasing inactive lifestyles, contributing to OW health-related 


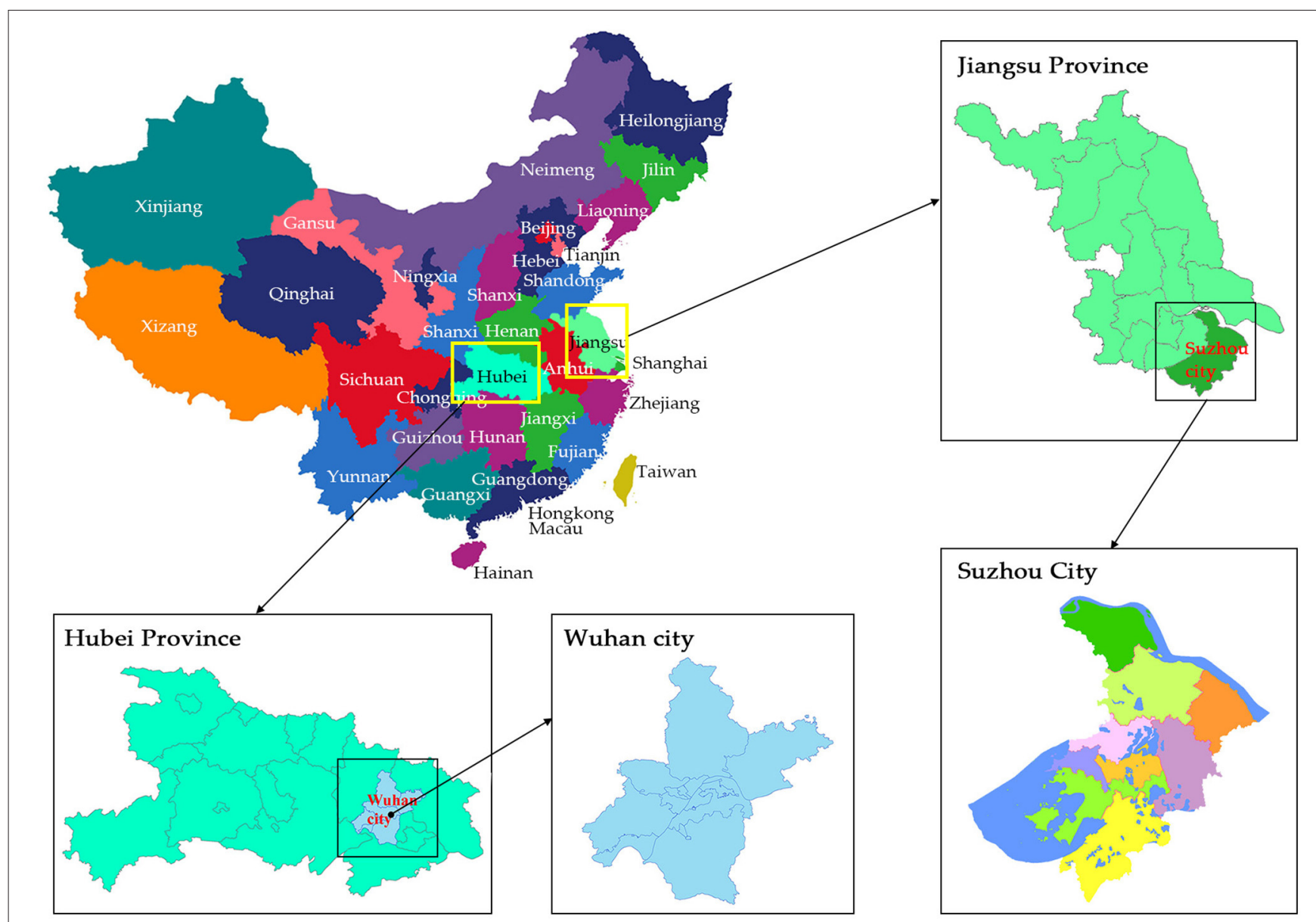

FIGURE 4 | Bootstrapping results with inner model $t$-values.

TABLE 7 | Mediation (indirect effect) result $(N=2,200)$.

\begin{tabular}{|c|c|c|c|c|c|c|c|}
\hline Hypotheses & OS/Beta & LL & UL & $T$ & $P$-values & Decision & Mediation \\
\hline $\mathrm{CP}->\mathrm{ER}->\mathrm{PH}$ & -0.035 & -0.054 & -0.053 & 3.830 & 0.001 & Significant & Partial \\
\hline $\mathrm{CP}->\mathrm{ER}->\mathrm{PsH}$ & -0.002 & -0.006 & 0.000 & 1.285 & 0.200 & Not significant & No mediation \\
\hline $\mathrm{CP}->\mathrm{ER}->\mathrm{OW}$ & -0.046 & -0.026 & -0.069 & 4.088 & 0.001 & Significant & Partial \\
\hline $\mathrm{CP}->\mathrm{ER}->\mathrm{PA}$ & -0.048 & -0.026 & -0.072 & 3.993 & 0.001 & Significant & Partial \\
\hline$L P->E R->P H$ & -0.235 & -0.279 & -0.195 & 10.766 & 0.001 & Significant & Partial \\
\hline LP $->$ ER $->$ PsH & 0.015 & -0.002 & 0.034 & 1.631 & 0.104 & Not significant & No mediation \\
\hline $\mathrm{LP}->\mathrm{ER}->\mathrm{OW}$ & -0.313 & -0.365 & -0.265 & 12.368 & 0.001 & Significant & Partial \\
\hline$L P->E R->P A$ & -0.323 & -0.374 & -0.274 & 12.639 & 0.001 & Significant & Partial \\
\hline
\end{tabular}

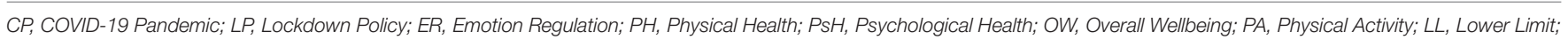
UL, Upper Limit.

problems (Krok and Zarzycka, 2020). The mediating effect of ER among the mediating paths was statistically significant as the $t$ values are higher than 1.96 and the $p$-values are $<0.05$. A study conducted in Mainland China reported that the effect of CP on emotional stability or the quality of life has negatively impacted China and many other parts of the world (Zhang and Ma, 2020). Our findings confirm that ER has a mediating effect because both lower and upper limits are negative values. The home confinement policy implementation regarding the containment of the COVID-19 negatively impacted the HRQoL (LipskayaVelikovsky, 2021). Similar results have been reported by Özdin and Bayrak Özdin (2020) and Rajkumar (2020) and found that ER, as a coping strategy, significantly impacts eliminating depression and anxiety. Cognitive ER as a coping strategy 
correlates with HRQoL (Dubey et al., 2020). ER strategies have a positive impact on psychological well-being (Extremera and Rey, 2014).

The closure of public spaces has negative impacts on the PA level. CP and LPs, directly and indirectly, impact socioeconomic, psychological, and physical health well-being aspects of the human society (Sang et al., 2021). The statistical analysis of our study revealed a significant negative relationship between the $\mathrm{CP}$ and PA, which means PA level decreased among the targeted research population of our study. PA levels decreased from local to global levels across the world due to the CP lockdown. PA is a natural preventive measure against different non-communicable diseases and plays a vital role in maintaining the HRQoL (Dai and Menhas, 2020; Sánchez Castillo et al., 2020). The decreasing PA and inactive lifestyle were identified as vital issues during home confinement (Bentlage et al., 2020). PA levels decreased from local to global levels across the world due to the CP lockdown. Playgrounds, public parks, and recreational spaces closed due to implementing the COVID-19 LP to cutoff the transmission of the virus. In similar findings by López-Sánchez et al. (2020), it was reported that PA levels declined from about 60.6 to $48.9 \%$ among the Spanish population.

The $\mathrm{PH}, \mathrm{PsH}$, and $\mathrm{OW}$ of the Chinese people were affected due to the $\mathrm{CP}$ and lockdown measures. It is suggested that PA is the most suitable preventive measure against chronic anxiety. In the context of PsH, the COVID-19 outbreak increased the mental health issues of Wuhan residents reported by Bao et al. (2020), and a high rate of depression prevalence among young Chinese people has been found during the lockdown period (Huang and Zhao, 2020). General well-being, $\mathrm{PH}$, and $\mathrm{PsH}$ are linked with PA. PA is also affected by the COVID-19 lockdown, negatively impacting the HRQoL. PA played a pivotal role in improving HRQoL, especially in the comorbidities reported by Hanke et al. (2020). In the context of PA related to our findings, similar results were reported in Greece and found adverse changes in PA due to the COVID-19 lockdown (Bourdas and Zacharakis, 2020). Our findings show that LP and PH, LP and PsH, LP and OW, and LP and PA also found statistically significant negative relationships as their $p$-values were $<0.05$. Similarly, a study conducted in Pakistan reported that the COVID-19 negatively influenced Pakistani students, further linked with high depression and mental anxiety (Salman et al., 2020). Likewise, a study conducted in Canada reported that outdoor and overall PA decreased due to the lockdown (Lesser and Nienhuis, 2020). In line with our findings, similar results have been reported by Narayanan et al. (2020) and found that the lifestyle of Indian people has been changed due to the COVID-19 lockdown measures, which further have negative impacts on HRQoL.

\section{CONCLUSIONS}

COVID-19 was declared as a global pandemic by the WHO after many cases across China were confirmed. Due to the severity of the COVID-19, many countries worldwide introduced different measures to contain the pathogen of the COVID-19, such as lockdown of the whole country, smart lockdown, social distancing, and body temperature monitoring at home confinement. The preventive measures taken by different countries had an impact on the socio-economic perspectives to HRQoL (Azizi et al., 2020). Our results show that the CP and the LPs negatively impact the HRQoL among the Chinese population. Additionally, the mediating role of ER was found to significantly improve the HRQoL, such as $\mathrm{CP}->\mathrm{ER}->\mathrm{PH}$, $\mathrm{CP} \rightarrow$ ER $->$ OW, CP $->$ ER $>>$ PA, LP $>>$ ER $->$ PH, LP $->$ ER $->$ OW, and LP $->$ ER $->$ PA. Stability and proper ER play a vital role in OW. PA is also important for OW, but due to the COVID-19 LPs across China, PA participation decreased. The mediating role of ER is critical for quality of life during the sudden emergence of an outbreak. ER can be defined as an individual's efforts to monitor and control their ecstatic response. It is suggested that ER intervention reduces the negative psychological impacts for improving quality of life. ER can function one's sentiments in their social environment effectively for quality of life.

\section{STUDY LIMITATIONS}

The study has several limitations. The cross-sectional study design is a major limitation of this study. Furthermore, to participate in the survey, the respondent must be 18 years old and literate. The convenience sampling technique was used under the non-probability sampling method according to the objective and nature of the study. The study results cannot be generalized to the whole population because it is hard to replicate the convenience sample results.

\section{DATA AVAILABILITY STATEMENT}

The datasets presented in this article are not readily available because the data belongs to a multi-country ongoing project. Requests to access the datasets should be directed to the corresponding author RM.

\section{ETHICS STATEMENT}

The studies involving human participants were reviewed and approved by the Ethics Committee of Soochow University (SUDA2020061H01) and the Ethics Committee of Wuhan Sports University (2020004). The study was conducted according to the Declaration of Helsinki's guidelines. The patients/participants provided their written informed consent to participate in this study.

\section{AUTHOR CONTRIBUTIONS}

JD is the principal investigator, while XS, YW, YC, and JH conducted an online survey and collected primary data. RM designed the study model, methodology, wrote the article, and did English editing. SK and MG guided psychological perspective. ZAS contributed to the discussion section with 
RM while MNA analyzed the data. BS, SM, and WI designed pictures, while XX did the proofreading and approved the manuscript. All authors contributed to the article and approved the submitted version.

\section{FUNDING}

This study is a part of Study on the Government Cultivation of Spontaneous Sports Organizations in China, funded by the

\section{REFERENCES}

Abbas, J., Mahmood, S., Ali, H., Ali, R. M., Ali, G., Aman, J., et al. (2019). The effects of corporate social responsibility practices and environmental factors through a moderating role of social media marketing on sustainable performance of business firms. Sustainability 11:3434. doi: 10.3390/su11123434

Al Zobbi, M., Alsinglawi, B., Mubin, O., and Alnajjar, F. (2020). Measurement method for evaluating the lockdown policies during the COVID-19 pandemic. Int. J. Environ. Res. Public Health 17:5574. doi: 10.3390/ijerph171 55574

Alwan, N. A., Burgess, R. A., Ashworth, S., Beale, R., Bhadelia, N., Bogaert, D., et al. (2020). Scientific consensus on the COVID-19 pandemic: we need to act now. Lancet. 396, e71-e72. doi: 10.1016/S0140-6736(20)32153-X

Anderson, J. C., and Gerbing, D. W. (1992). Assumptions and comparative strengths of the two-step approach: comment on Fornell and Yi. Sociol. Methods Res. 20, 321-333. doi: 10.1177/0049124192020003002

Anna, F. L. (2020). Travel Ban Goes Into Effect in Chinese City of Wuhan as Authorities Try to Stop Coronavirus Spread. Available online at: https://www. washingtonpost.com/world/asia_pacific/nine-dead-as-chinese-coronavirusspreads-despite-efforts- to-contain-it/2020/01/22/1eaade72-3c6d-11ea-afe2090eb37b60b1_story.html (accessed January 1, 2020).

Azizi, A., Achak, D., Aboudi, K., Saad, E., Nejjari, C., Nouira, Y., et al. (2020). Health-related quality of life and behavior-related lifestyle changes due to the COVID-19 home confinement: dataset from a Moroccan sample. Data Brief 32:106239. doi: 10.1016/j.dib.2020.106239

Bao, Y., Sun, Y., Meng, S., Shi, J., and Lu, L. (2020). 2019-nCoV epidemic: address mental health care to empower society. Lancet 395, e37-e38. doi: 10.1016/S0140-6736(20)30309-3

Bavel, J., Baicker, K., Boggio, P. S., Capraro, V., Cichocka, A., Cikara, M., et al. (2020). Using social and behavioural science to support COVID-19 pandemic response. Nat. Hum. Behav. 4, 460-471. doi: 10.1038/s41562-020-0884-z

Bentlage, E., Ammar, A., How, D., Ahmed, M., Trabelsi, K., Chtourou, H., et al. (2020). Practical recommendations for maintaining active lifestyle during the COVID-19 pandemic: a systematic literature review. Int. J. Environ. Res. Public Health 17:6265. doi: 10.3390/ijerph17176265

Bound, A. F. (2018). This "modern epidemic:" loneliness as an emotion cluster and a neglected subject in the history of emotions. Emot. Rev. 10, 242-254. doi: $10.1177 / 1754073918768876$

Bourdas, D. I., and Zacharakis, E. D. (2020). Impact of COVID-19 lockdown on physical activity in a sample of greek adults. Sports 8:139. doi: $10.3390 /$ sports 8100139

Brooks, S. K., Webster, R. K., Smith, L. E., Woodland, L., Wessely, S., Greenberg, N., et al. (2020). The psychological impact of quarantine and how to reduce it: rapid review of the evidence. Lancet 395, 912-920. doi: 10.1016/S0140-6736(20)30460-8

Chen, P., Mao, L., Nassis, G. P., Harmer, P., Ainsworth, B. E., and Li, F. (2020). Coronavirus disease (COVID-19): the need to maintain regular physical activity while taking precautions. J. Sport Health Sci. 9, 103-104. doi: 10.1016/j.jshs.2020.02.001

Cheng, S. K. W., Chong, G. H. C., Chang, S. S. Y., Wong, C. W., Wong, C. S. Y., Wong, M. T. P., et al. (2006). Adjustment to severe acute respiratory syndrome (SARS): Roles of appraisal and post-traumatic growth. Psychol. Health. 21, 301-317. doi: 10.1080/14768320500286450
National Social Science Fund of China (16BTY033, 2016.82021.12, PI: Daijianhui).

\section{ACKNOWLEDGMENTS}

We are very much thankful to all the researchers for data collection and processing. We thank all the survey participants in our study.
Chin, W. W. (2010). "How to write up and report PLS analyses," in Handbook of Partial Least Squares, eds V. Esposito Vinzi, W. Chin, J. Henseler, and H. Wang (Berlin, Heidelberg: Springer), 655-690. doi: 10.1007/978-3-54032827-8_29

Dai, J., and Menhas, R. (2020). Sustainable development goals, sports and physical activity: the localization of health-related sustainable development goals through sports in China: a narrative review. Risk Manag. Healthcare Pol. 13, 1419-1430. doi: 10.2147/RMHP.S257844

Duan, L., and Zhu, G. (2020). Psychological interventions for people affected by the COVID-19 epidemic. Lancet Psychiatry 7, 300-302. doi: 10.1016/S2215-0366(20)30073-0

Dubey, N., Podder, P., and Pandey, D. (2020). Knowledge of COVID19 and its influence on mindfulness, cognitive emotion regulation and psychological flexibility in the Indian Community. Front. Psychol. 11:589365. doi: 10.3389/fpsyg.2020.589365

Duy, B., and Yildiz, M. A. (2014). Adaptation of the regulation of emotions questionnaire (REQ) for adolescents. Turkish Psychol. Counseling Guidance J. 5, 23-35. Available online at: https://www.semanticscholar. org/paper/Adaptation-of-the-Regulation-of-Emotions-(REQ)-for-Duy/ c7c4161d2deedebb976b081ef3d2034ce319255b

Ekman, P. E., and Davidson, R. J. (1994). The Nature of Emotion: Fundamental Questions. Oxford: Oxford University Press.

Extremera, N., and Rey, L. (2014). Health-related quality of life and cognitive emotion regulation strategies in the unemployed: a cross-sectional survey. Health Qual. Life Outcomes 12:172. doi: 10.1186/s12955-014-0172-6

Götz, O., Liehr-Gobbers, K., and Krafft, M. (2010). "Evaluation of structural equation models using the partial least squares (PLS) approach," in Handbook of Partial Least Squares, eds V. Esposito Vinzi, W. Chin, J. Henseler, and H. Wang (Berlin, Heidelberg: Springer), 691-711. doi: 10.1007/978-3-540-32827-8_30

Goyal, K., Chauhan, P., Chhikara, K., Gupta, P., and Singh, M. P. (2020). Fear of COVID 2019: first suicidal case in India!. Asian J. Psychiatry 49:101989. doi: 10.1016/j.ajp.2020.101989

Gross, J. J. (1998). The emerging field of emotion regulation: an integrative review. Rev. Gen. Psycho. 2, 271-299. doi: 10.1037/1089-2680.2.3.271

Gross, J. J. (2013). Emotion regulation: taking stock and moving forward. Emotion 13, 359-365. doi: 10.1037/a0032135

Gross, J. J., and Munoz, R. F. (1995). Emotion regulation and mental health. Clin. Psychol. Sci. Pract. 2, 151-164. doi: 10.1111/j.1468-2850.1995.tb00036.x

Hair, J. F., Hult, G. T. M., Ringle, C. M., Sarstedt, M., and Thiele, K. O. (2017). Mirror, mirror on the wall: a comparative evaluation of composite-based structural equation modeling methods. J. Acad. Market. Sci. 45, 616-632. doi: 10.1007/s11747-017-0517-x

Haleem, A., and Javaid, M., Vaishya, R. (2020). Effects of COVID-19 pandemic in daily life. Curr. Med. Res. Pract. 3:11. doi: 10.1016/j.cmrp.2020.03.011

Hanke, A. A., Sundermeier, T., Boeck, H. T., Schieffer, E., Boyen, J., Braun, A. C., et al. (2020). Influence of officially ordered restrictions during the first wave of COVID-19 pandemic on physical activity and quality of life in patients after kidney transplantation in a telemedicine based aftercare program-A KTx $360^{\circ}$ sub study. Int. J. Environ. Res. Public Health 17:9144. doi: 10.3390/ijerph17239144

Hawkley, L. C., and Capitanio, J. P. (2015). Perceived social isolation, evolutionary fitness and health outcomes: a lifespan approach. Philos. Trans. Royal Soc. B Biol. Sci. 370:20140114. doi: 10.1098/rstb.2014.0114 
Henseler, J., Hubona, G., and Ray, P. A. (2016). Using PLS path modeling in new technology research: updated guidelines. Indus. Manag. Data Syst. 2015:382. doi: 10.1108/IMDS-09-2015-0382

Henseler, J., Ringle, C. M., and Sinkovics, R. R. (2009). The Use of Partial Least Squares Path Modeling in International Marketing. In New Challenges to International Marketing. Manchester: Emerald Group Publishing Limited. doi: 10.1108/S1474-7979(2009)0000020014

Holmes, E. A., O’Connor, R. C., Perry, V. H., Tracey, I., Wessely, S., Arseneault, L., et al. (2020). Multidisciplinary research priorities for the COVID-19 pandemic: a call for action for mental health science. Lancet Psychiatry 7, 547-560. doi: 10.1016/S2215-0366(20)30168-1

Hossain, M. M., Sultana, A., and Purohit, N. (2020). Mental health outcomes of quarantine and isolation for infection prevention: a systematic umbrella review of the global evidence. SSRN 2020:3561265. doi: 10.21203/rs.3.rs-25647/v1

Huang, Y., and Zhao, N. (2020). Generalized anxiety disorder, depressive symptoms and sleep quality during COVID-19 epidemic in China: a web-based cross-sectional survey. J. Med. Sci. 23, 15-19. doi: 10.21203/rs.3.rs-17172/v1

Julie,. B. (2020). Tips for Controlling Your Emotions During COVID-19. Available online at: https://firstthings.org/tips-for-controlling-your-emotions-duringcovid-19/ (accessed January 20, 2021).

Krok, D., and Zarzycka, B. (2020). Risk perception of COVID-19, meaningbased resources and psychological well-being amongst healthcare personnel: the mediating role of coping. J. Clin. Med. 9:3225. doi: 10.3390/jcm9103225

Leahy, R. L., Tirch, D., and Napolitano, L. A. (2011). Emotion Regulation in Psychotherapy: A Practitioner's Guide. Washington, DC: American Psychological Association.

Leppin, A., and Aro, A. R. (2009). Risk perceptions related to SARS and avian influenza: theoretical foundations of current empirical research. Int. J. Behav. Med. 16, 7-29. doi: 10.1007/s12529-008-9002-8

Lesser, I. A., and Nienhuis, C. P. (2020). The impact of COVID-19 on physical activity behavior and well-being of Canadians. Int. J. Environ. Res. Public Health 17:3899. doi: 10.3390/ijerph17113899

Li, J. B., Yang, A., Dou, K., Wang, L. X., Zhang, M. C., and Lin, X. (2021). Chinese public's knowledge, perceived severity, and perceived controllability of the COVID-19 and their associations with emotional and behavioural reactions, social participation, and precautionary behaviour: a national survey. PsyArXiv. doi: 10.31234/osf.io/5tmsh

Li, W., Yang, Y., Liu, Z. H., Zhao, Y. J., Zhang, Q., Zhang, L., et al. (2020). Progression of mental health services during the COVID-19 outbreak in China. Int. J. Biol. Sci. 16, 1732-1738. doi: 10.7150/ijbs.45120

Lin, Y., Zhong, P., and Chen, T. (2020). Association between Socioeconomic Factors and the COVID-19 outbreak in the 39 well-developed cities of China. Front. Public Health 8:546637. doi: 10.3389/fpubh.2020. 546637

Lipskaya-Velikovsky, L. (2021). COVID-19 isolation in healthy population in israel: challenges in daily life, mental health, resilience, and quality of life. Int. J. Environ. Res. Public Health 18:999. doi: 10.3390/ijerph180 30999

López-Sánchez, G. F., Pardhan, S., Trott, M., Sánchez-Castillo, S., Jackson, S. E., Tully, M., et al. (2020). The association between physical activity and cataracts among 17,777 people aged 15-69 years residing in Spain. Ophthal. Epidemiol. 27, 272-277. doi: 10.1080/09286586.2020.1730911

Low, R. S., Overall, N., Chang, V., and Henderson, A. M. (2020). Emotion regulation and psychological and physical health during a nationwide COVID19 lockdown. PsyArXiv. doi: 10.31234/osf.io/pkncy

Muzi, S., Sans,ò, A., and Pace, C. S. (2021). What's happened to Italian adolescents during the COVID-19 pandemic? A preliminary study on symptoms, problematic social media usage, and attachment: relationships and differences with pre-pandemic peers. Front. Psychiatry 12:590543. doi: $10.3389 /$ fpsyt.2021.590543

Narayanan, L., Pandit, M., Basu, S., Karmakar, A., Bidhan, V., Kumar, H., et al. (2020). Impact of lockdown due to COVID-19 outbreak: lifestyle changes and public health concerns in India. Preprints 2020060129. doi: 10.20944/preprints202006.0129.v1

Ozamiz-Etxebarria, N., Mondragon, N. I., Santamaría, M. D., and Gorrotxategi, M. P. (2020). Psychological symptoms during the two stages of lockdown in response to the COVID-19 outbreak: an investigation in a sample of citizens in Northern Spain. Front. Psychol. 11:1491. doi: 10.3389/fpsyg.2020. 01491

Özdin, S., and Bayrak Özdin, S. (2020). Levels and predictors of anxiety, depression and health anxiety during COVID-19 pandemic in Turkish society: the importance of gender. Int. J. Soc. Psychiatry 66, 504-511. doi: 10.1177/0020764020927051

Plümper, T., and Neumayer, E. (2020). Lockdown policies and the dynamics of the first wave of the Sars-CoV-2 pandemic in Europe. J. Eur. Public Pol. 27, 1-21. doi: 10.1080/13501763.2020.1847170

Qi, M., Li, P., Moyle, W., Weeks, B., and Jones, C. (2020). Physical activity, health-related quality of life, and stress among the Chinese adult population during the COVID-19 pandemic. Int. J. Environ. Res. Public Health 17:6494. doi: $10.3390 /$ ijerph17186494

Rajkumar, R. P. (2020). COVID-19 and mental health: a review of the existing literature. Asian J. Psychiatry 52:102066. doi: 10.1016/j.ajp.2020.102066

Ringle, C. M., Wende, S., and Becker, J. M. (2015). SmartPLS 3. Boenningstedt: SmartPLS; GmbH.

Rogowska, A. M., Pavlova, I., Kuśnierz, C., Ochnik, D., Bodnar, I., and Petrytsa, P. (2020). Does physical activity matter for the mental health of university students during the COVID-19 pandemic? J. Clin. Med. 9:3494. doi: $10.3390 /$ jcm 9113494

Rubin, G., Potts, H., and Michie, S. (2010). The impact of communications about swine flu (influenza A H1N1v) on public responses to the outbreak: results from 36 national telephone surveys in the UK. Health Technol. Assess 14, 183-266. doi: 10.3310/hta14340-03

Saarni, C. (2008). "The interface of emotional development with social context," in Handbook of emotions, eds M. Lewis, J. M. Haviland-Jones, and L. F. Barrett (The Guilford Press), 332-347. Available online at: https://psycnet.apa.org/ record/2008-07784-020

Salman, M., Asif, N., Mustafa, Z. U., Khan, T. M., Shehzadi, N., Tahir, H., et al. (2020). Psychological impairment and coping strategies during the COVID-19 pandemic among students in Pakistan: a cross-sectional analysis. Disast. Med. Public Health Preparedness 1, 1-22. doi: 10.1017/dmp.2020.397

Sánchez Castillo, S., Smith, L., Díaz Suárez, A., and López Sánchez, G. F. (2020). Analysis of physical activity and comorbidities in spanish asthmatics. Sustainability 12:5256. doi: 10.3390/su12135256

Sander, D., Armony, J., and Vuilleumier, P. (2013). The Cambridge Handbook of Human affective Neuroscience. Cambridge University Press. 5-3.

Sang, X., Menhas, R., Saqib, Z. A., Mahmood, S., Weng, Y., Khurshid, S., et al. (2021). The psychological impacts of COVID-19 home confinement and physical activity: a structural equation model analysis. Front. Psychol. 11:614770. doi: 10.3389/fpsyg.2020.614770

Saqib, Z. A., Dai, J., Rashid, M., Shahid, M., Karim, M., Sang, X., et al. (2020). Physical activity is a medicine for non-communicable diseases: a survey study regarding the perception of physical activity impact on health wellbeing. Risk Manag. Healthcare Pol. 13:2949. doi: 10.2147/RMHP.S2 80339

Shi, L., Lu, Z. A., and Que, J. Y. (2020). Prevalence of and risk factors associated with mental health symptoms among the general population in China during the coronavirus disease 2019 pandemic. J. Am. Med. Assoc. Netw. Open 3:14053. doi: 10.1001/jamanetworkopen.2020.14053

Sitlinger, A., and Zafar, S. Y. (2018). Health-related quality of life: the impact on morbidity and mortality. Surg. Oncol. Clin. North America 27, 675-684. doi: 10.1016/j.soc.2018.05.008

Sloan, E., Hall, K., Moulding, R., Bryce, S., Mildred, H., and Staiger, P. K. (2017). Emotion regulation as a transdiagnostic treatment construct across anxiety, depression, substance, eating and borderline personality disorders: a systematic review. Clin. Psychol. Rev. 57, 141-163. doi: 10.1016/j.cpr.2017.09.002

Tenenhaus, M., Vinzi, V. E., Chatelin, Y. M., and Lauro, C. (2005). PLS path modeling. Computat. Statist. Data Anal. 48, 159-205. doi: $10.1016 /$ j.csda.2004.03.005

Tsamakis, K., Triantafyllis, A. S., Tsiptsios, D., Spartalis, E., Mueller, C., Tsamakis, C., et al. (2020). COVID-19 related stress exacerbates common physical and mental pathologies and affects treatment. Exp. Therapeut. Med. 20, 159-162. doi: 10.3892/etm.2020.8671

Velotti, P., Civilla, C., Rogier, G., and Beomonte Zobel, S. (2021a). A fear of COVID-19 and PTSD symptoms in pathological personality: the mediating 
effect of dissociation and emotion dysregulation. Front. Psychiatry 12:590021. doi: 10.3389/fpsyt.2021.590021

Velotti, P., Rogier, G., Beomonte Zobel, S., Castellano, R., and Tambelli, R. (2021b). Loneliness, emotion dysregulation, and internalizing symptoms during coronavirus disease 2019: a structural equation modeling approach. Front. Psychiatry 11:581494. doi: 10.3389/fpsyt.2020.581494

Wang, C., Pan, R., Wan, X., Tan, Y., Xu, L., Ho, C. S., et al. (2020). Immediate psychological responses and associated factors during the initial stage of the 2019 coronavirus disease (COVID-19) epidemic among the general population in China. Int. J. Environ. Res. Public Health 17:1729. doi: 10.3390/ijerph17051729

Yang, Z. J. (2016). Altruism during Ebola: risk perception, issue salience, cultural cognition, and information processing. Risk Anal. 36, 1079-1089. doi: $10.1111 /$ risa. 12526

Zhang, Y., and Ma, Z. F. (2020). Impact of the COVID-19 pandemic on mental health and quality of life among local residents in Liaoning Province, China: a cross-sectional study. Int. J. Environ. Res. Public Health 17:2381. doi: $10.3390 /$ ijerph17072381
Conflict of Interest: The authors declare that the research was conducted in the absence of any commercial or financial relationships that could be construed as a potential conflict of interest.

Publisher's Note: All claims expressed in this article are solely those of the authors and do not necessarily represent those of their affiliated organizations, or those of the publisher, the editors and the reviewers. Any product that may be evaluated in this article, or claim that may be made by its manufacturer, is not guaranteed or endorsed by the publisher.

Copyright $\odot 2021$ Dai, Sang, Menhas, Xu, Khurshid, Mahmood, Weng, Huang, Cai, Shahzad, Iqbal, Gul, Saqib and Alam. This is an open-access article distributed under the terms of the Creative Commons Attribution License (CC BY). The use, distribution or reproduction in other forums is permitted, provided the original author(s) and the copyright owner(s) are credited and that the original publication in this journal is cited, in accordance with accepted academic practice. No use, distribution or reproduction is permitted which does not comply with these terms. 